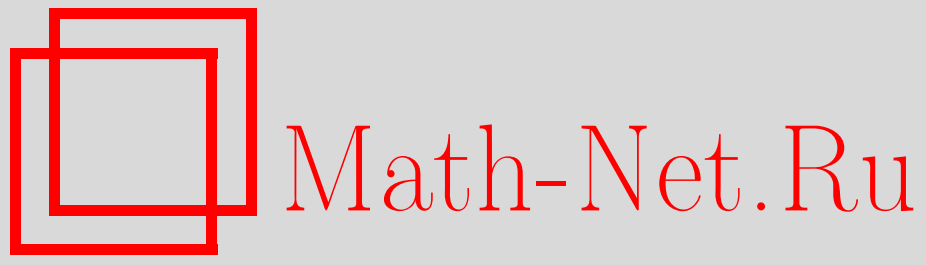

А. А. Панков, О нетривиальных решениях нелинейного уравнения Шрёдингера с магнитным полем, Функи. анализ и его прил., 2003, том 37, выпуск 1, 88-91

DOI: https://doi.org/10.4213/faa141

Использование Общероссийского математического портала MathNet.Ru подразумевает, что вы прочитали и согласны с пользовательским соглашением

http://www.mathnet.ru/rus/agreement

Параметры загрузки:

IP: 3.85 .7 .115

26 апреля 2023 г., 13:21:28

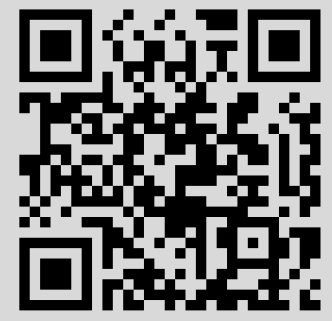


4. В заключении отметим, что спектральная теория операторов Шрёдингера с $\delta^{\prime}$-взаимодействием тесно связана со спектральной теорией неоднородно нагруженной струны, созданной М. Г. Крейном и И. С. Кацем [5-7]. Спектральная задача для неоднородной струны эквивалентна спектральной задаче для оператора Шрёдингера с $\delta^{\prime}$-взаимодействием с неотрицательной интенсивностью. Так, например, спектральная задача для однородной бесконечной струны с точечной массой $m$ на ней сводится к уравнению $u^{\prime \prime}+\lambda r(x) u(x)=0$, где $r(x)=1+m \delta(x)$ - плотность струны. Это уравнение эквивалентно уравнению $u^{\prime \prime}(x)+\lambda u(x)=0$ $(x \neq 0)$ и условиям сопряжения $u(+0)=u(-0), u^{\prime}(+0)-u^{\prime}(-0)=-\lambda m u(0)$. Подстановка $u^{\prime}=\psi(x)$ сводит эту задачу к уравнению $-\psi^{\prime \prime}(x)=\lambda \psi(x)(x \neq 0)$ и условиям сопряжения $\psi^{\prime}(+0)=\psi^{\prime}(-0), \psi(+0)-\psi(-0)=m \psi^{\prime}(0)$, т. е. к задаче для оператора Шрёдингера с одноточечным $\delta^{\prime}$-взаимодействием интесивности $m$.

Для включения общего $\delta^{\prime}$-взаимодействия в теорию струны необходимо в последней рассматривать и случай отрицательных масс. В созданной полной и законченной спектральной теории неоднородной струны свойство неотрицательности масс существенно как для метода, так и для формулировки основных результатов.

\title{
ЛИТЕРАТУРА
}

1. Albeverio S., Gesztesy F., Høegh-Krohn R., Holden H. Solvable models in quantum mechanics. Springer-Verlag, Berlin, 1988. 2. Triebel H. Fractals and spectra. Birkhaüser, Basel-Boston-Berlin, 1997. 3. Глазман И. М. Прямые методы качественного спектрального анализа сингулярных дифференциальных операторов. Наука, M., 1967. 4. Albeverio S., Nizhnik L. P. Укр. матем. журн., 52 (5), 582-589 (2000). 5. Гохберг И. Ц., Крейн М. Г. Теория вольтерровых операторов в гильбертовом пространстве и ее приложения. Наука, М., 1967. 6. Кац И. С., Крейн М. Г. Изв. вузов, матем., 3, № 2, 136-153 (1958). 7. Каи И. С. Докл. АН СССР, 164, №5, 975-978 (1965).

\section{О нетривиальных решениях нелинейного уравнения Шрёдингера с магнитным полем}

\author{
(C) 2003. А. А. ПАнков
}

0. Рассмотрим стационарное нелинейное магнитное уравнение Шрёдингера

$$
\left(\frac{1}{\mathrm{i}} \nabla+A(x)\right)^{2} u+V(x) u=g(x,|u|) u, \quad x \in \mathbb{R}^{n} .
$$

Это уравнение описывает амплитуду стоячих волн $\psi(x, t)=\exp (\mathrm{i} \omega t) \cdot u(x)$ для соответствующего эволюционного нелинейного уравнения Шрёдингера с магнитным полем. Мы интересуемся (комплексными) решениями уравнения (1), исче- 
зающими на бесконечности. Для дальнейшего удобно записать уравнение (1) в несколько ином виде:

$$
\left(\frac{1}{\mathrm{i}} \nabla+A(x)\right)^{2} u+V(x) u=\alpha f(x, u), \quad x \in \mathbb{R}^{n},
$$

где $\alpha= \pm 1$. В сделанных далее предположениях $\alpha=+1$ отвечает случаю притягивающего самовзаимодействия, в то время как $\alpha=-1$ означает, что потенциал самовзаимодействия отталкивающий.

1. Всюду ниже предполагается, что $V$ и $A$ вещественны и выполнены следующие условия (i)-(v).

(i) Левая часть формуль (2) определяет существенно самосопряженный полуограниченный снизу оператор на $C_{0}^{\infty}\left(\mathbb{R}^{n}\right)$.

Это условие выполнено, если, например, электрический потенциал $V$ лежит в $L_{\mathrm{loc}}^{\infty}\left(\mathbb{R}^{n}\right)$ и полуограничен снизу, а магнитный потенциал $A$ локально липшицев [1]. Обозначим через $H$ соответствующий самосопряженный оператор в $L^{2}\left(\mathbb{R}^{n}\right)$, а через $E$ область определения замкнутой квадратичной формы $q_{H}$, порожденной оператором $H(E-$ гильбертово пространство с естественным скалярным произведением).

(ii) Спектр $\sigma(H)$ оператора $H$ дискретен.

Если $V$ полуограничен снизу, то из справедливости условия (ii) для $A=0$ следует его справедливость для любого $A$ (см. [2]). Другие достаточные условия дискретности спектра можно найти в [3], а необходимые и достаточные - в [4].

Пусть $F: \mathbb{R}^{n} \times \mathbb{C}=\mathbb{R}^{n} \times \mathbb{R}^{2} \rightarrow \mathbb{R}$ удовлетворяет условию Каратеодори и $F(x, \cdot) \in C^{1}\left(\mathbb{R}^{2}\right)$ для почти всех $x \in \mathbb{R}^{n}$. Пусть $f(x, u)=\nabla_{u} F(x, u)$. Положим $2^{*}=2 n /(n-2)$ при $n \geqslant 3$ и $2^{*}=+\infty$ в противном случае.

(iii) Существуют такие константы $c>0$ и $p \in\left(2,2^{*}\right)$, ито для почти всех $x \in \mathbb{R}^{n}$

(iv) $f(x, 0)=0 u$

$$
|F(x, u)| \leqslant c\left(1+|u|^{p}\right), \quad u \in \mathbb{C} .
$$

$$
\lim _{u \rightarrow 0} \operatorname{ess} \sup _{\mathbb{R}^{n}}\left|\frac{f(x, u)}{u}\right|=0 .
$$

(v) Cуществует такое $q>2$, ито для почти всех $x \in \mathbb{R}^{n} u \quad u \neq 0$

$$
0<q F(x, u) \leqslant f(x, u) \cdot \bar{u} .
$$

Типичный пример: $f(x, u)=|u|^{p-2} u, p \in\left(2,2^{*}\right)$.

Отметим, что уравнение (1) инвариантно относительно естественного действия окружности $S^{1}$. В более общем уравнении (2) мы не требуем такой инвариантности.

Очевидно, что $u=0$ - решение уравнения (2). Поэтому в дальнейшем мы интересуемся только нетривиальными решениями.

2. Функционал

$$
\Phi_{\alpha}(u)=\frac{\alpha}{2} q_{H}(u)-\int_{\mathbb{R}^{n}} F(x, u) \mathrm{d} x
$$

корректно определен на $E \subset H^{1}\left(\mathbb{R}^{n}\right)$ и принадлежит классу $C^{1}$. Его критические точки - в точности слабые решения уравнения (2) в $E$. Отметим, что, в отличие 
от ситуации с функционалом $\Phi_{+1}$, отрицательное подпространство квадратичной части функционала $\Phi_{-1}$ бесконечномерно.

Лемма. Функционал $\Phi_{\alpha}$ удовлетворяет условию Пале-Смейла, т. е. любая последовательность $u_{k} \in E$, такая, что $\Phi_{\alpha}\left(u_{k}\right)$ ограничено $и \nabla \Phi_{\alpha}\left(u_{k}\right) \rightarrow 0$, относительно компактна в $E$.

Теорема 1. Если $\alpha=+1$, то уравнение (2) имеет нетривиальное решение в Е. Если $\alpha=-1$, то (2) имеет нетривиальное решение тогда и только тогда, когда $\inf \sigma(H)<0$.

Рассмотрим случай нечетной нелинейности: $f(x,-u)=-f(x, u)$ (эквивалентно, $F(x,-u)=F(x, u)$ ). Тогда появляются пары решений $\pm u$ (орбиты антиподального действия на $E$ группы $\left.\mathbb{Z}_{2}\right)$.

Теорема 2. Пусть нелинейность $f$ нечетна. Если $\alpha=+1$, то существует последовательность $\pm u_{k} \in E$ пар решении уравнения (2) с критическими значениями $c_{k}^{(+1)}=\Phi_{+1}\left(u_{k}\right) \rightarrow+\infty$. Если $\alpha=-1$, то число нетривиальных пар решений уравнения (2) не меньше числа отрицательных собственных значений оператора $H$.

Если нелинейность имеет вид $f(x, u)=g(x,|u|) u$, то $\Phi_{\alpha}$ инвариантен относительно действия окружности $S^{1}$ на $E$ умножением. В этом случае утверждение теоремы 2 сохраняет силу с заменой $\mathbb{Z}_{2}$-орбит (пар) решений $S^{1}$-орбитами.

3. Прямое вычисление показывает, что для критической точки $u$

$$
2 \Phi_{\alpha}(u)=\int_{\mathbb{R}^{n}}[f(x, u) \cdot \bar{u}-2 F(x, u)] \mathrm{d} x .
$$

Следовательно, нетривиальные критические точки должны иметь положительные критические значения. В условиях теоремы 1 с $\alpha=+1$ нетривиальная критическая точка может быть найдена с помощью теоремы о горном перевале, если $\inf \sigma(H)>0$, и теоремы о зацеплении в противном случае (см. $[5,6])$. Если же $\alpha=-1$, то функционал $\Phi_{\alpha}$ ограничен сверху. Так как $\Phi_{\alpha}$ удовлетворяет условию Пале-Смейла, то он достигает своего максимального значения $\sup \Phi_{\alpha} \geqslant 0$. При этом $\sup \Phi_{\alpha}=0$ тогда и только тогда, когда $\inf \sigma(H) \geqslant 0$.

В случае теоремы 2 воспользуемся вариантом идеи теоремы о фонтане [7]. Пусть $\lambda_{1} \leqslant \lambda_{2} \leqslant \cdots \leqslant \lambda_{k} \leqslant \cdots-$ собственные значения оператора $H$ с учетом кратности, а $e_{k}, k \in \mathbb{N},-$ соответствующие линейно независимые собственные функции. Обозначим через $E_{k}^{(+1)}$ и $E_{k}^{(-1)}$ замкнутые подпространства в $E$, порожденные функциями $\left\{e_{1}, \ldots, e_{k}\right\}$ и $\left\{e_{j}\right\}_{j \geqslant k}$ соответственно. Отметим, что $E_{k}^{(+1)}$ конечномерно, а $E_{k}^{(-1)}$ имеет конечную коразмерность (основное техническое различие между случаями $\alpha=+1$ и $\alpha=-1$ ).

Можно показать, что для любого $k \in \mathbb{N}$ существует такое $r_{k}^{(\alpha)}$, что $\Phi_{\alpha}(u) \leqslant 0$ при $u \in E_{k}^{(\alpha)},\|u\| \geqslant r_{k}^{(\alpha)}$. Пусть $B_{k}^{(\alpha)}=\left\{u \in E_{k}^{(\alpha)}:\|u\| \leqslant r_{k}^{(\alpha)}\right\}$. Обозначим через $\Gamma_{k}^{(\alpha)}$ множество всех гомеоморфизмов $\varphi: B_{k}^{(\alpha)} \rightarrow E$ вида $\varphi=\mathrm{id}+\psi$, где $\psi-$ компактное отображение, таких, что $\varphi=\mathrm{id}$ на $\partial B_{k}^{(\alpha)}$ и $\varphi(-u)=-\varphi(u)$ (условие компактности излишне в случае $\alpha=+1)$. Положим

$$
c_{k}^{(\alpha)}=\inf _{\varphi \in \Gamma_{k}^{(\alpha)}} \sup _{u \in B_{k}^{(\alpha)}} \Phi_{\alpha}(\varphi(u)) .
$$


Можно показать, что $c_{k}^{(\alpha)}$ больше или равно 0 и является критическим значением функционала $\Phi_{\alpha}$. Очевидно также, что $\alpha c_{k}^{(\alpha)} \leqslant \alpha c_{k+1}^{(\alpha)}$. Оказывается, что $c_{k}^{(+1)} \rightarrow$ $+\infty$, а $c_{k}^{(-1)}>0$ в том и только том случае, когда $\lambda_{k}<0$. (Для оценки снизу критических значений $c_{k}^{(\alpha)}$ используется вариант техники, предложенной в [8].) Наконец, если $c_{k}^{(\alpha)}=c_{k+1}^{(\alpha)}=c>0$, то функционал $\Phi_{\alpha}$ имеет бесконечно много критических точек на уровне $c$.

4. Насколько известно автору, имеется лишь несколько работ [9-12], посвященных уравнению (2). Во всех этих работах предполагается, что $H>0$, а нелинейность притягивающая $(\alpha=+1)$. При различных предположениях установлено существование нетривиального решения. При этом в $[9,12]$ не требуется дискретности спектра $\sigma(H)$, а в $[10,11]$ налагаются более сильные условия, чем дискретность спектра. Кроме того, в случае постоянных магнитного поля и потенциала $V$ в [9] получены условия существования бесконечного числа решений. В работах $[10,11]$ изучается также квазиклассическое поведение решений.

Намного больше известно об уравнении $(2)$ без магнитного поля $(A=0)$. Отметим здесь лишь результаты, непосредственно связанные с теоремами 1 и 2. (Во всех случаях имеется в виду только притягивающая нелинейность. Случай отталкивающей нелинейности, по-видимому, не рассматривался.) Существование нетривиального решения установлено в [13] в предположении, что $V(x) \geqslant 0$ и $V(x) \rightarrow \infty$ при $x \rightarrow \infty$. В [14] условие на рост потенциала было несколько ослаблено и в случае нечетной нелинейности доказано существование бесконечного числа решений. Наконец, в [8] отмечено, что в этих результатах достаточно предполагать дискретность спектра оператора $H$. Кроме того, в [8] получены некоторые результаты о кратности решений в случае, когда $H$ имеет конечное число (положительных) собственных значений ниже существенного спектра.

Автор признателен рецензенту за полезные замечания.

\section{ЛИТЕРАТУРА}

1. Leinfelder H., Simader C. Math. Z., 176, 1-19 (1981). 2. Avron J., Herbst I., Simon B. Duke Math. J., 45, 847-883 (1978). 3. Kondratiev V., Shubin M. Comm. Partial Differential Equations, 27, 477-525 (2002). 4. Kondratiev V., Maz'ya V., Shubin M. arXiv: math.SP/0206140, 2002. 5. Rabinowitz P. Minimax methods in critical point theory with applications to differential equations. Amer. Math. Soc., Providence, R.I., 1986. 6. Willem M. Minimax theorems. Birkhäuser, Boston, 1996. 7. Bartsch T. Nonlin. Analysis, 20, 1205-1216 (1993). 8. Bartsch T., Pankov A., Wang Z.-Q. Comm. Contemp. Math., 3, 549-569 (2001). 9. Esteban E. M., Lions P. L. In: Partial differential equations and calculus of variations. V. I. Birkhäuser, Boston, 1989, pp. 401-449. 10. Kurata K. Nonlin. Analysis, 41, 763-778 (2000). 11. Cingolani S., Secchi S. arXiv: math.AP/0107047, 2001. 12. Schindler I., Tintarev K. Rostock. Math. Kolloq., 56, 49-54 (2002). 13. Rabinowitz P. Z. Angew. Math. Phys., 43, 270-291 (1992). 14. Bartsch T., Wang Z.-Q. Comm. Partial Differential Equations, 20, 1725-1741 (1995).

Винницкий государственный педагогический университет

Поступило в редакцию 5 марта 2002 г, 